\title{
Treatment With Statin on Atheroma Regression Evaluated by Intravascular Ultrasound With Virtual Histology (TRUTH Study)
}

Rationale and Design

\author{
Tsuyoshi Nozue, MD ${ }^{1}$; Shingo Yamamoto, MD²; Shinichi Tohyama, MD³; Shigeo Umezawa, MD; \\ Tomoyuki Kunishima, MD ${ }^{5}$; Akira Sato, $\mathrm{MD}^{6}$; Shogo Miyake, $\mathrm{MD}^{7}$; Youichi Takeyama, $\mathrm{MD}^{8}$; \\ Yoshihiro Morino, MD9; Takao Yamauchi, MD ${ }^{10}$; Toshiya Muramatsu, MD ${ }^{11}$; \\ Kiyoshi Hibi, MD ${ }^{12}$; Takashi Sozu, $\mathrm{PhD}^{13}$; Ichiro Michishita, MD ${ }^{1}$ \\ on Behalf of Kanagawa PTCA Conference Study Group
}

\begin{abstract}
Background Many clinical trials have shown that 3-hydroxy-3-methylglutaryl-conenzyme A reductase inhibitors (statins) can significantly reduce the incidence of coronary artery disease in both primary and secondary prevention. A recent study showed that aggressive lipid-lowering therapy with statins could achieve regression of coronary artery plaque evaluated with gray-scale intravascular ultrasound (IVUS). However, the actual changes in coronary artery plaque composition produced by statin therapy have not been well delineated.

Methods and Results This study will be a prospective, open-label, randomized multicenter study of 160 patients with stable or unstable angina who have undergone percutaneous coronary intervention with Virtual Histology ${ }^{\mathrm{TM}}$ IVUS (VH-IVUS). Patients will be randomly assigned to either the pitavastatin or pravastatin group. After treatment for 24-40 weeks, VH-IVUS will be performed again in the same segment of the coronary artery. The primary endpoint will be quantitative changes in each of the 4 components measured by VH-IVUS.

Conclusion The treatment with statin on atheroma regression evaluated by intravascular ultrasound with Virtual Histology (TRUTH) study will be the first multicenter study using VH-IVUS to evaluate the effects of statins on changes in coronary artery plaque composition and the findings will clarify the mechanisms of coronary artery plaque stabilization. (Circ J 2009; 73: 352-355)
\end{abstract}

Key Words: Coronary artery disease; Plaque composition; Statins; Virtual Histology ${ }^{\mathrm{TM}}$ intravascular ultrasound (VH-IVUS)

$\mathbf{T}$ he 3-hydroxy-3-methylglutaryl-conenzyme A (HMG$\mathrm{CoA})$ reductase inhibitors (statins) revolutionized the treatment of hypercholesterolemia, and many clinical trials have shown that they can significantly reduce

(Received June 18, 2008; revised manuscript received September 11 , 2008; accepted October 8, 2008; released online December 26, 2008) ${ }^{1}$ Division of Cardiology, Department of Internal Medicine, Yokohama Sakae Kyosai Hospital, ${ }^{2}$ Department of Cardiology, Tsurumi Nishiguchi Hospital, ${ }^{3}$ Department of Cardiology, Kanagawa Cardiovascular and Respiratory Center, Yokohama, ${ }^{4}$ Department of Cardiology, Hiratsuka Kyosai Hospital, Hiratsuka, ${ }^{5}$ Fourth Department of Internal Medicine, Mizonokuchi Hospital, Teikyo University School of Medicine, Kawasaki, ${ }^{6}$ Cardiovascular Center, Yokosuka Kyosai Hospital, Yokosuka, ${ }^{7}$ Department of Cardiology, Ebina General Hospital, Ebina, ${ }^{8}$ Division of Cardiology, Showa University Fujigaoka Hospital, Yokohama, ${ }^{9}$ Division of Cardiology, Tokai University School of Medicine, Isehara, ${ }^{10}$ Department of Cardiology, Tokyo Women's Medical University, Tokyo, ${ }^{11} D e-$ partment of Cardiology, Saiseikai Yokohama City Eastern Hospital, ${ }_{12}^{12}$ Division of Cardiology, Yokohama City University Medical Center, Yokohama and ${ }^{13}$ The Center for Advanced Medical Engineering and Informatics, Osaka University, Suita, Japan

Mailing address: Ichiro Michishita, MD, Division of Cardiology, Department of Internal Medicine, Yokohama Sakae Kyosai Hospital, Federation of National Public Service Personnel Mutual Associations, 132 Katsura-cho, Sakae-ku, Yokohama 247-8581, Japan. E-mail: yk411015@fsinet.or.jp

All rights are reserved to the Japanese Circulation Society. For permissions, please e-mail: cj@j-circ.or.jp the incidence of coronary artery disease (CAD) in both primary and secondary prevention ${ }^{1-5}$ Furthermore, intensive lipid-lowering therapy significantly reduces the risk of coronary events compared with moderate lipid-lowering therapy 6,7

Imaging studies of coronary artery plaque have contributed considerably to our understanding of the benefits of intensive lipid-lowering therapies. The REVERSAL trial ${ }^{8}$ evaluated the effects of statin on coronary artery plaque volume using gray-scale intravascular ultrasound (IVUS). Moderate lipid-lowering therapy with $40 \mathrm{mg}$ of pravastatin did not halt plaque progression, but aggressive lipid-lowering therapy with $80 \mathrm{mg}$ of atorvastatin did. The ASTEROID trial investigated the effect of aggressive lipid-lowering therapy with $40 \mathrm{mg}$ of rosuvastatin on regression of coronary artery plaque, and in Japan, 10-20 mg of atorvastatin prevented further progression of coronary atherosclerosis ${ }^{10}$ while the ESTABLISH study showed a regression of coronary artery plaque with $20 \mathrm{mg}$ of atorvastatin!1 Further study has been performed in a Japanese population, using IVUS to evaluate the effects of rosuvastatin on regression of coronary atherosclerosis ${ }^{12}$ However, those trials have only evaluated quantitative changes of coronary artery plaque using gray-scale IVUS.

Virtual Histology ${ }^{\mathrm{TM}}$ IVUS (VH-IVUS) (Volcano Therapeutics, Inc, Rancho Cordova, CA, USA) has been devel- 


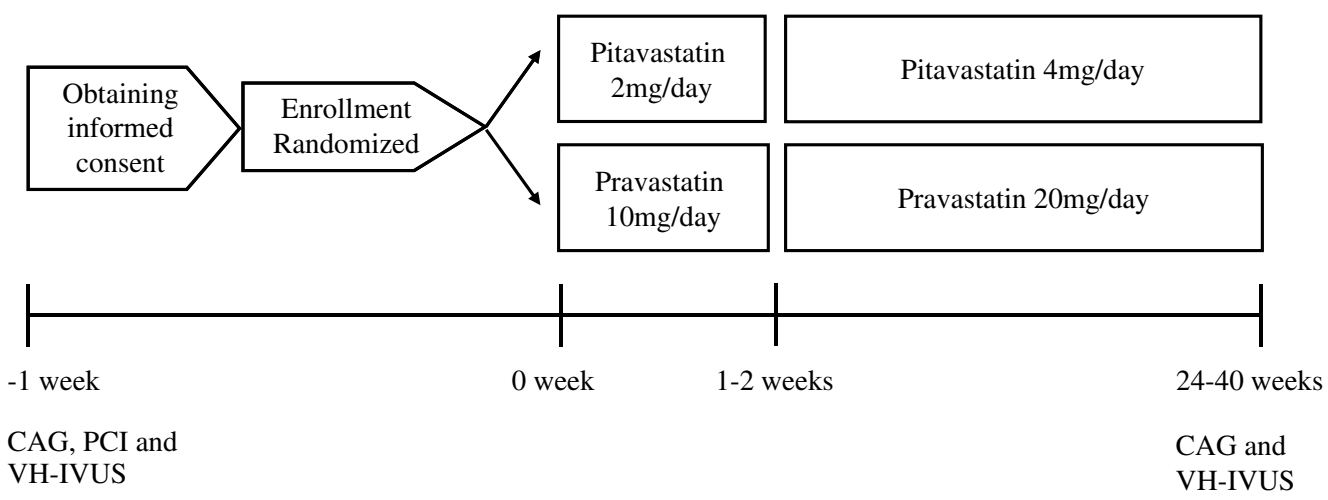

Fig 1. Flow chart of the study timeline. CAG, coronary angiography; PCI, percutaneous coronary intervention; VHIVUS, Virtual Histology ${ }^{\mathrm{TM}}$ intravascular ultrasound.

oped by the Cleveland Clinic! ${ }^{3}$ It captures and analyzes radiofrequency ultrasound signals and can provide realtime automated maps by classifying atherosclerotic plaque into the 4 basic tissue types of fibrous, fibro-fatty, densecalcium, and necrotic-core. The accuracy of VH-IVUS is approximately $80-92 \%$ in vitro ${ }^{13}$ and approximately $87.1-$ $96.5 \%$ in vivo ${ }^{14}$

Pitavastatin has a powerful lipid-lowering effect and has been used in Japan since 2003 where its clinical efficacy and safety have been demonstrated for patients with hypercholesterolemia. ${ }^{15}$ Takashima et al used IVUS to demonstrate that $2 \mathrm{mg}$ of pitavastatin induced significant coronary artery plaque regression associated with a significant reduction of low-density lipoprotein (LDL)-cholesterol (C) 16 To confirm their findings, the Japan Assessment of Pitavastatin and Atorvastatin in Acute Coronary Syndrome (JAPANACS) trial was performed to evaluate the effects of pitavastatin and atorvastatin on coronary artery plaque regression in patients with acute coronary syndrome (ACS) ${ }^{17}$ However, the effects are evaluated by gray-scale IVUS and it is unknown whether plaque composition or vulnerability was changed.

Because it is unclear whether there are changes in the composition of coronary artery plaque following statin therapy, the present study has been designed to use VHIVUS to examine the effects of statins. Furthermore, 2 statins (pitavastatin and pravastatin) will be evaluated. As placebo-controlled trials of statins are no longer ethically acceptable, a comparator group receiving pravastatin will be included. Pravastatin was selected because the Japanese MEGA study found that its use contributed to the prevention of CAD! 18

\section{Methods}

\section{Study Design}

The treatment with statin on atheroma regression evaluated by intravascular ultrasound with Virtual Histology (TRUTH) study will be a prospective, open-label, randomized multicenter study of 160 patients with stable or unstable angina who have undergone successful percutaneous coronary intervention (PCI) of the culprit lesion under IVUS guidance and have a plaque area $\geq 50 \%$ proximal or distal to the PCI site. In addition, patients must have baseline LDL-C level $\geq 140 \mathrm{mg} / \mathrm{dl}$ or judged by a physician to require treatment with statins to manage coronary risk factors. Exclusion criteria include: (1) established treat- ment with statins, (2) hepatic disorder, or AST (GOT) or ALT (GPT) greater than 2.5-fold the upper limit of normal, (3) renal dysfunction (serum creatinine $\geq 2.0 \mathrm{mg} / \mathrm{dl}$ ), (4) history of a severe muscular disorder or $\mathrm{CK}(\mathrm{CPK})$ greater than 2.5-fold the upper limit of normal, (5) acute myocardial infarction within $24 \mathrm{~h}$ of enrollment, (6) failed PCI, (7) cerebrovascular accident within 1 month of enrollment, (8) concurrent or a history of malignant tumor within 5 years, (9) receiving LDL apheresis, (10) homozygous familial hypercholesterolemia, (11) established treatment with any of the following: cyclosporine, fibrate, nicotinic acid, colestyramine and probucol, or (12) ineligible in the opinion of the investigator.

The included patients will give written informed consent approved by the institutional ethical committee after PCI and then be randomly assigned to either the pitavastatin or pravastatin group. The randomization will be stratified by the status of their CAD (stable or unstable angina) and by institution. The supervising physician will administer the allocated drugs within 1 week of PCI with a starting dose of pitavastatin $2 \mathrm{mg}$ /day or pravastatin $10 \mathrm{mg}$ /day for the first 1-2 weeks. After safety evaluation, the dose will be increased $4 \mathrm{mg} /$ day or $20 \mathrm{mg} /$ day, respectively. The patients will continue taking the allocated drugs until the end of study, until certain endpoints have occurred, or when they decide to discontinue participation in this study. IVUS, coronary angiography (CAG) and laboratory determination will be performed at baseline and 24-40 weeks (Fig 1).

The enrollment of patients is planned between December 2005 and February 2008 or until enrollment has been completed. The planned study duration is December 2005 to December 2008. The study has been registered at University Hospital Medical Information Network (UMIN) (UMIN ID: C000000311).

\section{IVUS Examination}

IVUS will be performed at baseline after culprit lesion intervention and at the 24-40 week visit. IVUS data will be derived from the region of interest (ROI), defined as $>5 \mathrm{~mm}$ distal to the stent (ROI 1) and/or $>5 \mathrm{~mm}$ proximal to the stent (ROI 2), with a reproducible index such as side branches. After injection of $0.2 \mathrm{mg}$ nitroglycerin, a $20-\mathrm{MHz}$, 2.9-F phase-array IVUS catheter (Eagle Eye Gold, Volcano Therapeutics) will be placed distal to ROI 1 and/or ROI 2. Pullback will be performed automatically at $0.5 \mathrm{~mm} / \mathrm{s}$ to the proximal part of ROI. Exclusion criteria include: (1) bypass graft at the site of PCI, (2) previous PCI lesion at 
the site where IVUS evaluation is planned, (3) IVUS plaque area $<50 \%$ at a non-PCI site ( $>5 \mathrm{~mm}$ proximal or distal to the PCI site), and (4) lesion is ineligible in the opinion of the investigator.

\section{IVUS Analysis}

IVUS images recorded in DVD format will be analyzed at the core laboratory to assess the changes in volume of each of the 4 components measured by VH-IVUS and the quantitative changes of the coronary artery measured by gray-scale IVUS (ie, vessel area and volume, lumen area and volume, plaque area and volume).

\section{Endpoints}

The primary endpoint will be quantitative changes in each of the 4 plaque components measured by VH-IVUS.

The secondary endpoint will be quantitative changes in the coronary artery parameters measured by gray-scale IVUS (vessel area and volume, lumen area and volume, plaque area and volume) and evaluation of major adverse cardiac events (MACE) [cardiac death, Q-wave infarction, non-Q-wave infarction, target lesion revascularization and coronary artery bypass graft]. Furthermore, changes in serum lipid levels [total cholesterol (TC), LDL-C, triglyceride (TG), high-density lipoprotein (HDL)-C], plasma total platelet-activating factor acetylhydrolase (PAF-AH) and HDL-associated PAF-AH concentrations, high-sensitivity C-reactive protein (hs-CRP), lipoprotein(a) [Lp(a)], oxidized LDL (Ox-LDL), apolipoprotein (apo) AI and apo $B$ will be evaluated.

\section{Safety Monitoring}

Safety will be evaluated at $1-2,4,8,12,16,20$, and 2440 weeks after enrollment. The Assessment Committee of Effect will evaluate MACE and any other adverse events.

\section{Sample Size Calculation}

The main goal of this trial is to evaluate differences between 2 statins in terms of changes in the composition of coronary artery plaque measured by VH-IVUS, according to the impact of differences in lipid-lowering effects. At present, there is no information in the literature about the effects of statins on the composition of coronary artery plaque. Therefore, it is difficult to set the target number of patients to achieve the primary endpoint. The study group estimated the necessary sample size based on the assumption that changes in plaque composition are accompanied by changes in plaque volume. The percent change in plaque volume was estimated by a linear relationship between the percent change of plaque volume and LDL-C that was reported by Okazaki et al!1 According to this formula, the percent change of plaque volume was $-11.5 \%$ in the pitavastatin group and $-5.3 \%$ in the pravastatin group accompanied by respective changes in LDL-C of $-47.0 \%$ and $-29.8 \%$. Okazaki et al also reported that the standard deviation of the percent changes in plaque volume after statin therapy was $12.8 \%$. Assuming these factors, the sample size required in this study is 69 patients in each group to achieve $80 \%$ power by a 2 -sided 2 -sample t-test at a significance level of 5\%. Assuming 10\% of patients drop out, the sample size per group will be $69 / 0.9=77$ patients.

\section{Data Management}

Patient information, blood samples, and IVUS images will be coded with the identification number of this study and the coded number for individual identification will remain blinded. Blood samples will be obtained after overnight fasting. Serum lipids, apolipoproteins, hs-CRP, Lp(a) and Ox-LDL levels will be measured at a central clinical laboratory (SRL Inc, Tokyo, Japan). Serum TC, LDL-C, HDL-C and TG levels will be measured by standard enzymatic methods. Ox-LDL levels will be measured by an enzyme immunoassay as previously described 19,20 Serum Lp(a) levels will be measured by a latex-enhanced turbidimetric immunoassay. Total PAF-AH and HDL-associated PAF-AH concentrations will be measured by an enzymelinked immunosorbent assay according to previously described methods (BML Inc, Tokyo, Japan) ${ }^{21}$ IVUS and CAG images will be analyzed at the core laboratory.

\section{Statistical Analysis}

Primary Endpoints Changes in composition of coronary artery plaque measured by VH-IVUS will be evaluated using analysis of covariance including the baseline measurement as a covariate. When an adjustment for multiplicity of testing is considered, a closed testing procedure in which the test for each component of composition is performed in the order of necrotic-core, fibro-fatty, fibrous, dense-calcium, will be applied with a significance level of $5 \%$. An analysis adjusting for factors of dynamic allocation will be performed.

Secondary Endpoints Quantitative changes in the coronary artery parameters measured by gray-scale IVUS (vessel area and volume, lumen area and volume, plaque area and volume) will be evaluated by a 2 -sided 2 -sample t-test.

\section{Study Organization}

Principal Investigator Ichiro Michishita, Division of Cardiology, Department of Internal Medicine, Yokohama Sakae Kyosai Hospita, Yokohama, Japan.

Biostatistician Takashi Sozu, The Center for Advanced Medical Engineering and Informatics, Osaka University, Suita, Japan.

Core Laboratory Mitsuyasu Terashima, Cardio Vascular Imaging Center (CVIC), Toyohashi, Japan.

Assessment Committee of Effect Kiyoshi Hibi, Yokohama City University Medical Center, Yokohama, Japan.

\section{Conclusion}

The TRUTH study will be the first multicenter study using VH-IVUS to evaluate the effects of statins on the composition of coronary artery plaque. The group is investigating the relationship between changes in plaque composition and lipid levels in stable or unstable angina patients receiving lipid-lowering therapy with pitavastatin or pravastatin. Furthermore, the study group hopes to show the process of coronary artery plaque stabilization by statins and that these findings will clarify the mechanisms of prevention of CAD by statin therapy.

\section{Acknowledgment}

This study is supported by a grant from the Japan Heart Foundation.

\section{References}

1. Scandinavian Simvastatin Survival Study Group. Randomized trial of cholesterol lowering in 4444 patients with coronary heart disease: The Scandinavian Simvastatin Survival Study (4S). Lancet 1994; 344: $1383-1389$.

2. Shepherd J, Cobbe SM, Ford I, Isles CG, Lorimer AR, MacFarlane 
PW, et al. Prevention of coronary heart disease with pravastatin in men with hypercholesterolemia: West of Scotland Coronary Prevention Study Group. N Engl J Med 1995; 333: 1301-1307.

3. Byington RP, Jukema JW, Salonen JT, Pitt B, Bruschke AV, Hoen H, et al. Reduction in cardiovascular events during pravastatin therapy: Pooled analysis of clinical events of the Pravastatin Atherosclerosis Intervention Program. Circulation 1995; 92: 2419-2425.

4. The Long-Term Intervention with Pravastatin in Ischaemic Disease (LIPID) Study Group. Prevention of cardiovascular events and death with pravastatin in patients with coronary heart disease and a broad range of initial cholesterol levels. N Engl J Med 1998; 339: 13491357.

5. Sacks FM, Pfeffer MA, Moye LA, Rouleau JL, Rutherford JD, Cole TG, et al. The effect of pravastatin on coronary events after myocardial infarction in patients with average cholesterol levels. $N$ Engl $J$ Med 1996; 335: 1001-1009.

6. Cannon CP, Braunwald E, McCabe CH, Rader DJ, Rouleau JL, Belder R, et al; Pravastatin or Atorvastatin Evaluation and Infection Therapy-Thrombolysis in Myocardial Infarction 22 Investigators. Intensive versus moderate lipid lowering with statins after acute coronary syndromes. N Engl J Med 2004; 350: 1495-1504.

7. LaRosa JC, Grundy SM, Waters DD, Shear C, Barter P, Fruchart JC, et al; Treating to New Targets (TNT) Investigators. Intensive lipid lowering with atorvastatin in patients with stable coronary disease. $N$ Engl J Med 2005; 352: 1425-1435.

8. Nissen SE, Tuzcu EM, Schoenhagen P, Brown BG, Ganz P, Vogel RA, et al; REVERSAL Investigators. Effect of intensive compared with moderate lipid-lowering therapy on progression of coronary atherosclerosis: A randomized controlled trial. JAMA 2004; 291: $1071-1080$.

9. Nissen SE, Nicholls SJ, Sipahi I, Libby P, Raichlen JS, Ballantyne $\mathrm{CM}$, et al; ASTEROID Investigators. Effect of very high-intensity statin therapy on regression of coronary atherosclerosis: The ASTEROID trial. JAMA 2006; 295: 1556-1565.

10. Yamada T, Azuma A, Sasaki S, Sawada T, Matsubara H; REACH Study Group. Randomized evaluation of atorvastatin in patients with coronary heart disease: A serial intravascular ultrasound study. Circ $J$ 2007; 71: $1845-1850$.

11. Okazaki S, Yokoyama T, Miyauchi K, Shimada K, Kurata T, Sato H, et al. Early statin treatment in patients with acute coronary syndrome: Demonstration of the beneficial effect on atherosclerotic lesions by serial volumetric intravascular ultrasound analysis during half a year after coronary event: The ESTABLISH Study. Circulation 2004; 110: $1061-1068$

12. Takayama T, Hiro T, Yamagishi M, Daida H, Saito S, Yamaguchi T, et al. Rationale and design for a study using intravascular ultrasound to evaluate effects of rosuvastatin on coronary artery atheroma in Japanese subjects: COSMOS study (Coronary Atherosclerosis Study Measuring Effects of Rosuvastatin Using Intravascular Ultrasound in Japanese Subjects). Circ J 2007; 71: 271-275.

13. Nair A, Kuban BD, Tuzcu EM, Schoenhagen P, Nissen SE, Vince DG. Coronary plaque classification with intravascular ultrasound radiofrequency data analysis. Circulation 2002; 106: 2200-2206.

14. Nasu K, Tsuchikane E, Katoh O, Vince DG, Virmani R, Surmely JF, et al. Accuracy of in vivo coronary plaque morphology assessment: A validation study of in vivo virtual histology compared with in vitro histopathology. J Am Coll Cardiol 2006; 47: 2405-2412.

15. Kajinami K, Takekoshi N, Saito Y. Pitavastatin: Efficacy and safety profiles of a novel synthetic HMG-CoA reductase inhibitor. Cardiovasc Drug Rev 2003; 21: 199-215.

16. Takashima H, Ozaki Y, Yasukawa T, Waseda K, Asai K, Wakita Y, et al. Impact of lipid-lowering therapy with pitavastatin, a new HMGCoA reductase inhibitor, on regression of coronary atherosclerotic plaque. Circ J 2007; 71: 1678-1684.

17. Miyauchi K, Kimura T, Morimoto T, Nakagawa Y, Yamagishi M, Ozaki Y, et al. Japan assessment of pitavastatin and atorvastatin in acute coronary syndrome (JAPAN-ACS): Rationale and design. Circ J 2006; 70: $1624-1628$.

18. Nakamura H, Arakawa K, Itakura H, Kitabatake A, Goto Y, Toyota T, et al; MEGA Study Group. Primary prevention of cardiovascular disease with pravastatin in Japan (MEGA Study): A prospective randomized controlled trial. Lancet 2006; 368: 1155-1163.

19. Itabe H, Yamamoto H, Imanaka T, Shimamura K, Uchiyama H, Kimura J, et al. Sensitive detection of oxidatively modified low density lipoprotein using a monoclonal antibody. J Lipid Res 1996; 37: $45-53$.

20. Kohno H, Sueshige N, Oguri K, Izumidate H, Masunari T, Kawamura $\mathrm{M}$, et al. Simple and practical sandwich-type enzyme immunoassay for human oxidatively modified low density lipoprotein using antioxidized phosphatidylcholine monoclonal antibody and antihuman apolipoprotein-B antibody. Clin Biochem 2000; 33: 243-253.

21. Kujiraoka T, Iwasaki T, Ishihara M, Ito M, Nagano M, Kawaguchi A, et al. Altered distribution of plasma PAF-AH between HDLs and other lipoproteins in hyperlipidemia and diabetes mellitus. J Lipid Res 2003; 44: 2006-2014.

\section{Appendix 1}

\section{Investigators}

Ichiro Mizuguchi, Taku Iwaki, Shimpei Ito, Kenichi Kobori, Yuki Ishibashi, Hideki Ouchi, Manabu Takai, Division of Cardiology, Department of Internal Medicine, Yokohama Sakae Kyosai Hospital; Naoto Yoshizawa, Department of Cardiology, Tsurumi Nishiguchi Hospital; Kazuki Fukui, Masayuki Nakao, Takeshi Nakagawa, Tomoyori Nakatogawa, Yasuo Ohkusu, Hiroyuki Ozaki, Kei Hatori, Junya Hosoda, Shingo Kato, Department of Cardiology, Kanagawa Cardiovascular and Respiratory Center; Akihiko Niwa, Masayuki Igawa, Yuko Onishi, Isshi Kobayashi, Takayuki Onishi, Hidetoshi Suzuki, Yoko Kato, Department of Cardiology, Hiratsuka Kyosai Hospital; Toshihiro Nozato, Hiroyuki Hikita, Hirokazu Oohigashi, Cardiovascular Center, Yokosuka Kyosai Hospital; Kentaro Takato, Ryoma Shibue, Department of Cardiology, Ebina General Hospital; Hiroshi Suzuki, Tokutada Satoh, Fuyuki Asano, Nobuyuki Shimizu, Kohei Wakabayashi, Yuuki Honda, Division of Cardiology, Showa University Fujigaoka Hospital; Yuji Ikari, Hiroki Takeuchi, Division of Cardiology, Tokai University School of Medicine; Naoya Fujita, Department of Cardiology, Tokyo Women's Medical University; Yoshiaki Ito, Department of Cardiology, Saiseikai Yokohama City Eastern Hospital. 